\title{
Unicystic Ameloblastoma with Mural Proliferation
}

\author{
İlknur Eninanç ${ }^{1}$, Defne Yalçın Yelerr ${ }^{1}$, Halit Şengel ${ }^{2}$, Hasan Yeler ${ }^{2}$, Ömer Fahrettin Göze ${ }^{3}$
}

Departments of ${ }^{1}$ Oral and Maxillofacial Radiology and ${ }^{2}$ Oral and Maxillofacial Surgery, Faculty of Dentistry; ${ }^{3}$ Department of Pathology, Medical Faculty, Sivas Cumhuriyet University, Sivas, Turkey.

\section{Corresponding Author: \\ Dr İlknur Eninanç \\ Email: i.eninanc2@gmail.com}

This is an Open Access article distributed under the terms of the Creative Commons Attribution License (creativecommons.org/ licenses/by/3.0).

Received : January 10, 2021

Accepted : March 19, 2021

Published : May 30, 2021

\begin{abstract}
Background: Unicystic ameloblastoma (UA) is a rare variant of ameloblastoma and represents about $6 \%$ of all ameloblastomas. Radiologically, it is very similar to odontogenic jaw cysts due to its unilocular radiolucency. Case Report: This report discusses the clinical, radiological, histopathological and surgical findings of mural unicystic ameloblastoma occupying the right maxillary sinus on a background of dentigerous cyst associated with an impacted tooth in a 22-year-old female patient. Conclusion: It is very uncommon to see this lesion in the maxilla since it usually involves the posterior region of the mandible. Differentiation of an UA from a dentigerous cyst is radiographically difficult when UA is associated with an impacted tooth. Among all UA types, UA with mural proliferation has the highest rate of recurrence. Long-term follow-up for recurrence should be considered for patients with UA.
\end{abstract}

Keywords: Ameloblastoma, Dentigerous Cyst, Mandible, Maxilla, Odontogenic Cysts.

\section{Introduction}

Ameloblastoma is a benign but locally aggressive odontogenic tumor [1]. In 2005, the World Health Organization (WHO) classified ameloblastomas as solid/multicystic, extraosseous/ peripheral, desmoplastic and unicystic types [2]. Unicystic ameloblastoma (UA) is a rare type of ameloblastoma which accounts for approximately $6 \%$ of all ameloblastomas [3]. Histologically, UA exhibits an ameloblastic epithelium that occupies a cystic space [4]. It may result from reduced enamel epithelium, from a dentigerous cyst or due to solid ameloblastomas that undergo cystic degeneration [5]. UA generally affects people between the ages of 11 and 30 and has equal sex distribution [6]. It is more commonly found in the mandible $[4,6]$. While small lesions are asymptomatic, larger lesions may cause facial asymmetry and ulceration of the overlying mucosa [7]. Radiologically, UA closely resembles odontogenic jaw cysts due to its unilocular radiolucency [3]. With its clinical and radiographic features mimicking a cyst, UA is considered to be a less aggressive variant of ameloblastoma and responds more favorably to conservative treatment $[3,4]$.

This case report discusses the clinical, radiological, histopathological and surgical findings of a mural unicystic ameloblastoma with an atypical location, filling the right maxillary sinus in a background of dentigerous cyst together with an impacted tooth.

\section{Case Report}

A 22-year-old female with no systemic disease presented to the Oral and Maxillofacial Radiology clinic with complaints of swelling and mild pain in her face in the previous month. On clinical extraoral examination, facial asymmetry due to swelling of the right maxillary sinus region was noted and intraoral examination showed swelling of the right alveolar region [Fig.1]. Overlying mucosa appeared smooth and intact. Palpable lymph nodes were absent. 
A panoramic radiograph and CBCT (cone beam computed tomography) scan revealed a radiolucent and unilocular lesion $(40 \times 31 \times 38 \mathrm{~mm})$ with well-demarcated borders occupying the entire right maxillary sinus in the presence of an impacted tooth (No. 18). Root resorption and mobility were observed in the teeth (No. 15, 16 and 17) closely associated with the lesion. As was evident from a panoramic radiograph taken from the patient two years back, the lesion had displaced the impacted tooth to the maxillary sinus over time [Fig.2]. The patient reported that she had no signs or symptoms 2 years ago when the panoramic radiograph was taken and there were no noticeable pathologic lesions at that time.

The affected region was aspirated, resulting and a yellow (straw colored) fluid containing cholesterol crystals. While a dentigerous cyst was considered as a preliminary diagnosis, UA was also kept in mind as a differential diagnosis. Initially, marsupialization was performed due to the large size of the lesion. The impacted tooth (No.18), the primary cause of the lesion, was extracted along with mobile teeth and a drain was placed in the extraction socket [Fig.3].

Biopsy was performed, the specimens $(n=6)$ were processed, H\&E (hematoxylin-eosin) stained and examined under light microscope. The tissue fragments sent for histopathologic examination appeared soft, gray-white colored and gelatinous macroscopically and the largest was $2.5 \times 1.5 \times 1.5 \mathrm{~cm}$ in size. Sections were prepared from the biopsy material for microscopic examination. The sections of the largest specimen showed formation of a dense collagenous connective tissue underneath the multilayered spongiotic squamous epithelium lining the dentigerous cyst, clusters of inflammatory cells (lymphocytes and histiocytes), microcalcifications and small, isolated ameloblastic cell trabeculae. In a separate section, a lesion was observed in the continuation of the surface epithelium which was characterized by round nodular ameloblastic cell

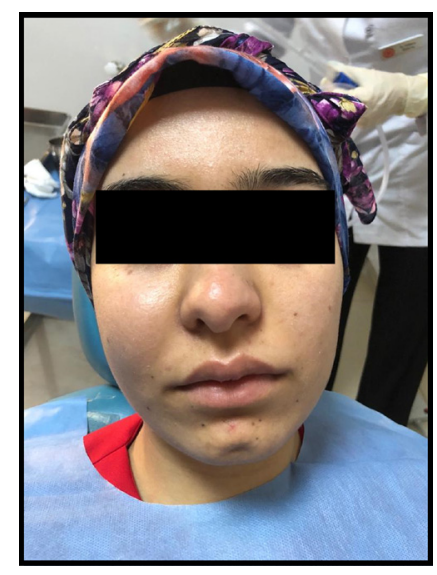

Fig.1: Facial asymmetry due to swelling of the right maxillary sinus.

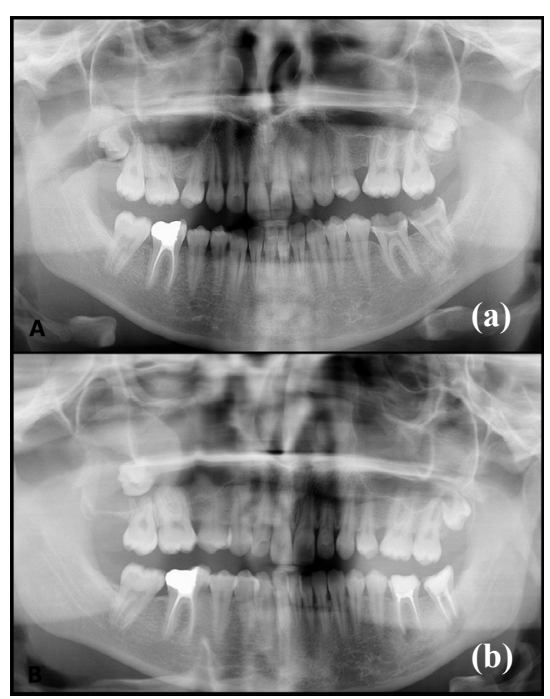

Fig.2(a): Panoramic radiograph of the patient taken 2 years ago; (b): Radiograph showing the lesion.

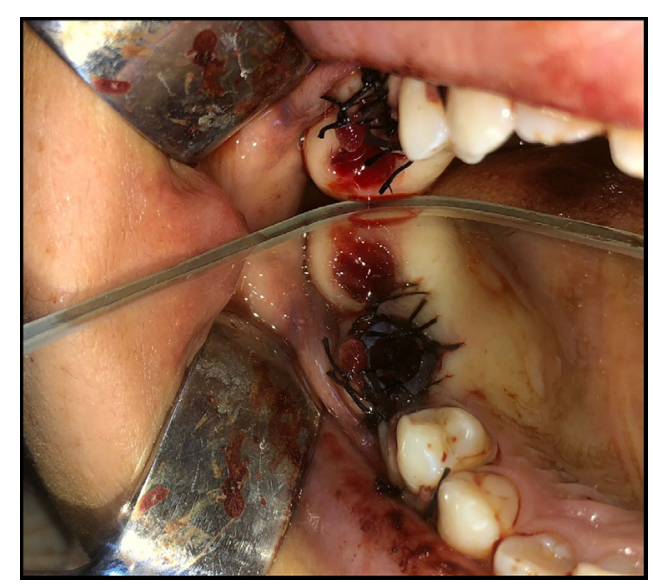

Fig.3: Drain placed in the affected region. 
groups facing the lumen and islands of ameloblastic cells embedded in the wall. The final diagnosis of mural unicystic ameloblastoma on a background of dentigerous cyst was established on the basis of these findings [Fig.4].

Initially, marsupialization was performed. Six months after marsupialization, the lesion was found to move away from lateral nasal wall and orbital floor and totally enucleated. New bone formation from the periphery to the center and improvement were observed on CBCT images obtained at 2, 6, 12 and 24 months following enucleation [Fig.5]. Additionally, improvement in the alveolar area was noticed on an intraoral examination of the patient 5 months after enucleation [Fig.6]. The patient has been followed for 2.5 years with no post-operative complications or recurrence.

\section{Discussion}

Unicystic ameloblastoma is mostly found in the mandibular ramus and posterior region of the maxilla is considered as an atypical and rare location for UA [3]. Investigators reported that $90 \%$ of the cases involve the mandible with the majority are located in the molar and ramus regions [8]. In the currently presented case, the lesion which was associated with the impacted tooth (No.18) occupied the entire maxillary sinus and caused mild pain, facial asymmetry and root resorption in the affected teeth.

A dentigerous cyst and other odontogenic cysts, odontogenic keratocysts, giant cell granuloma, odontogenic myxoma and ossified fibroma may be considered in the differential diagnosis of ameloblastoma. When UA is associated with an unerupted tooth, it is difficult to distinguish it from a dentigerous cyst radiographically [9]. In the current case, the presence of an impacted tooth together with the lesion and aspiration of a straw colored fluid from the area led to consideration of a dentigerous cyst as preliminary diagnosis.

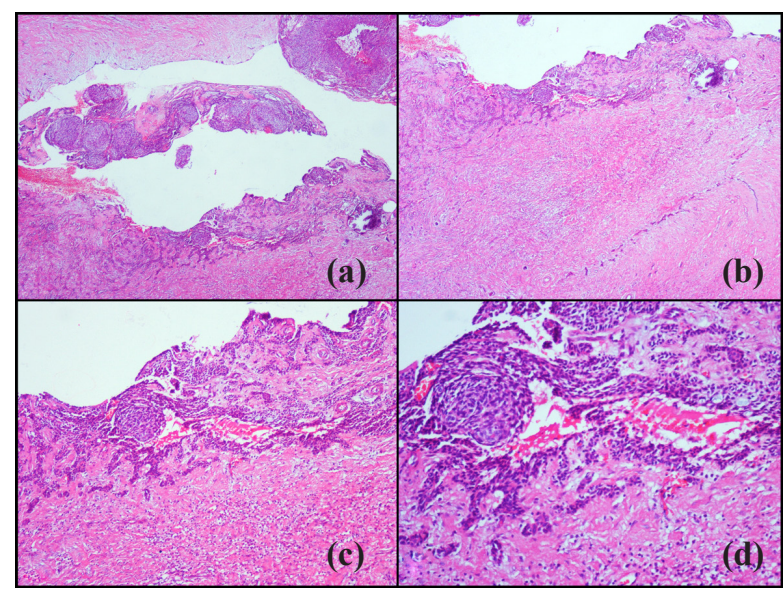

Fig.4(a): General appearance of mural unicystic ameloblastoma. $H E \times 4$; (b): Dentigerous cyst epithelium on the surface and round ameloblastic proliferation in one zone and strands of ameloblastic cells within the wall in the deeper layer HE $\times$ 4; (c): Cyst epithelium and ameloblastic proliferation in one zone - detail HE $\times$ 10; (d): Goblet cells and adjacent ameloblastic proliferation in the cyst epithelium- detail. $H E \times 20$.

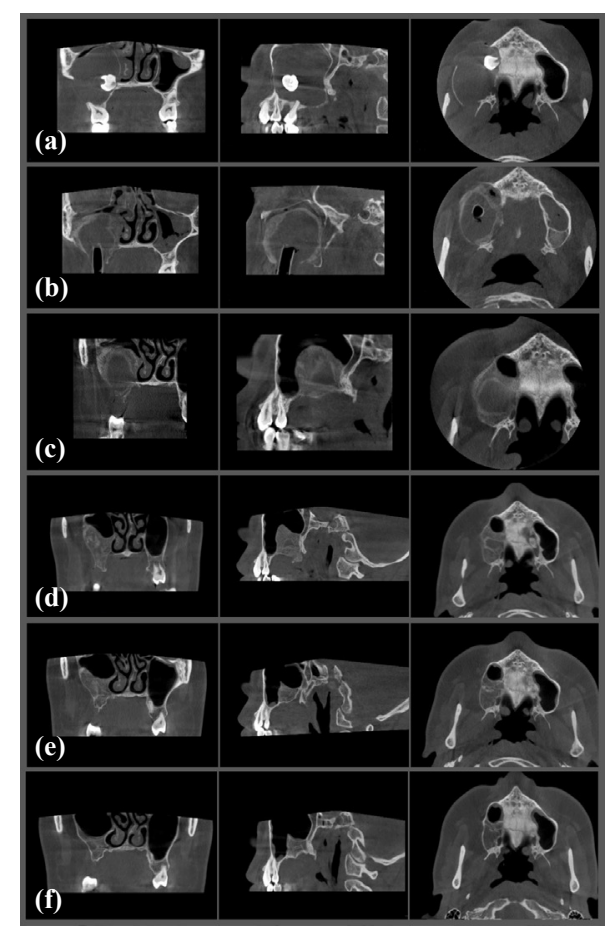

Fig.5(a): $C B C T$ scan showing the lesion associated with an impacted tooth, occupying the entire right maxillary sinus; (b): Reduced size of the lesion at 3 months after marsupialization; (c): $C B C T$ image at 2 months after enucleation; (d): $C B C T$ image at 6 months after enucleation; (e): $C B C T$ image at 12 months after enucleation; (f): $C B C T$ image at 24 months after enucleation. 
Histologically, UA is classified into 3 subtypes: luminal, intraluminal and mural. Among these subtypes, the mural type has the highest rate of recurrence due to its potential to penetrate and breach the fibrous wall and invade the cancellous bone adjacent to the epithelium [10]. Although UAs are regarded as a less aggressive form of ameloblastoma, the mural form of UA has a high tendency to recur, with one study reporting a recurrence rate of $35 \%[11,12]$. Therefore, the use of less aggressive treatment modalities are recommended to prevent recurrence and a repeat surgery unless UA lesions are widespread or cause aesthetic problems in younger patients [13].

Marsupialization is a more conservative form of treatment that is employed for odontogenic cystic lesions to reduce the size of the lesion and limit the extent of surgery [14]. It has been used as the primary treatment of large odontogenic cystic lesions for several years [15]. Marsupialization is effective for reducing the tumor size in both unicystic ameloblastoma and multicystic ameloblastoma. However, the speed of shrinkage was observed to be significantly faster for unicystic ameloblastoma than that of multicystic ameloblastoma when treated with marsupialization [16]. Lau and Samman [17] found a recurrence rate of $18 \%$ for unicystic ameloblastomas after marsupialization and reported that marsupialization followed by curettage might reduce the recurrence rate. UA has a more favorable response to enucleation or curettage than solid or multicystic ameloblastomas [18]. Moreover, since the maxillary bone has a spongy structure, maxillary ameloblastomas may behave more aggressively than mandibular ameloblastomas clinically [19]. Consistently, the currently presented lesion located at an anatomic space, the maxillary sinus, has progressed over a short term. Marsupialization was considered as the initial intervention because of the proximity of the lesion to anatomic structures, followed by enucleation 6 months later. The patient has been followed for 2.5 years for the risk of recurrence due

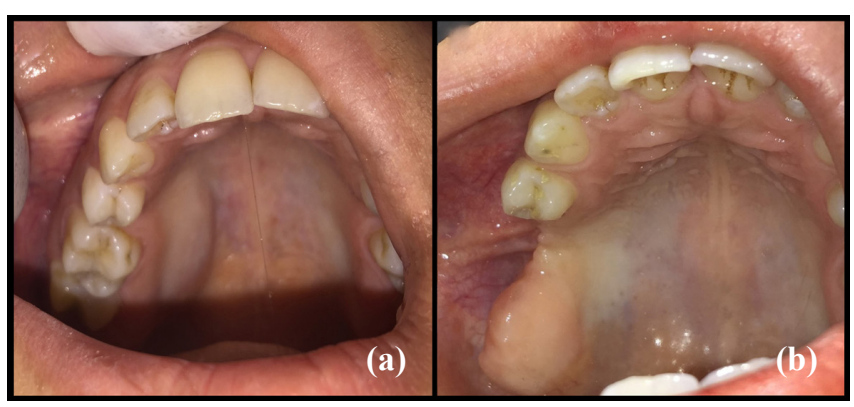

Fig.6: Intraoral examination showing (a): Swelling in the right alveolar region at presentation (b): Improvement at 5 months after enucleation.

to the presence of mural proliferation. Improvement was observed in the maxillary sinus area which started to assume its original morphology over time. The patient is currently being followed, without any signs of recurrence.

\section{Conclusion}

Unicystic ameloblastomas can be seen atypically in the maxillary sinus region. Unicystic ameloblastoma should also be included in the differential diagnosis of unilocular radiolucencies because it resembles a dentigerous cyst both clinically and radiographically. This allows selection of the most appropriate treatment. Because of the risk of recurrence associated with the mural type, post-operative radiographic examinations should be performed at frequent intervals and longterm follow-up of the patients is warranted. Early diagnosis and treatment are important to preserve more bone tissue.

Contributors: IE: manuscript writing, patient management; DYY, HS: manuscript editing, patient management; HY, OFG: critical inputs into the manuscript. IE will act as a study guarantor. All authors approved the final version of this manuscript and are responsible for all aspects of this study. Funding: None; Competing interests: None stated.

\section{References}

1. Sciubba JJ, Fantasia JA, Kahn LB, editors, Benign odontogenic tumors. In: Atlas of tumor pathologytumors and cysts of the jaw. Washington, DC: AFIP; 1999. pp. 71-85. 
2. Barnes L, Eveson JW, Reichart P, Sidransky D, World Health Organization Classification of Tumors, Pathology and Genetics. Head and Neck Tumors, IARC Press, Lyon, France; 2005. pp.296-300.

3. Philipsen HP, Reichard PA. Unicystic ameloblastoma. A review of 193 cases from literature. Oral Oncol. 1998;34:317-325.

4. Paikkatt VJ, Sreedharan S, Kannan VP. Unicystic ameloblastoma of the maxilla: A case report. J Indian Soc Pedod Prev Dent. 2007;25:106-110.

5. Figueiredo NR, Meena M, Dinkar AD, Malik S, Khorate $\mathrm{M}$. Unicystic ameloblastoma presenting as a multilocular radiolucency in the anterior mandible: A Case Report. J Dent Res Dent Clin Dent Prospect. 2015;9:199-204.

6. Singh A, Shaikh S, Samadi FM, Shrivastava S, Verma R. Maxillary unicystic ameloblastoma: A review of the literature. Natl J Maxillofac Surg. 2011;2:163-168.

7. Kumar KRK, George GB, Padiyath S, Rupak S. Mural unicystic ameloblastoma crossing the midline: a rare case report. Int J Odontostomat. 2012;6(1):97-103.

8. Li TJ, Wu YT, Yu SF, Yu GY. Unicystic ameloblastoma: A clinicopathologic study of 33 Chinese patients. Am J Surg Pathol. 2000;24:1385-1392.

9. White SC, Pharoah MJ. Oral radiology: Principles and interpretation. $7^{\text {th }}$ ed. St. Louis: Mosby/Elsevier; 2014.

10. Li TJ, Kitano M, Arimura K, Sugihara K. Recurrence of unicystic ameloblastoma: a case report and review of the literature. Arch Pathol Lab Med. 1998;122:371-374.

11. Chaudhary Z, Sangwan V, Pal US, Sharma P. Unicystic ameloblastoma: A diagnostic dilemma. Natl J Maxillofac Surg. 2011;2:89-92.
12. Mahadesh J, Rayapati DK, Maligi PM, Ramachandra P. Unicystic ameloblastoma with diverse mural proliferation - A hybrid lesion. Imaging Sci Dent. 2011;41:29-33.

13. Panneerselvam K, Kavitha B, Panneerselvam E, Parameswaran A. Mural unicystic ameloblastoma mimicking odontogenic cyst. Journal of Family Medicine and Primary Care. 2020;9(5):2524-2527.

14. Nakamura N, Higuchi $Y$, Tashiro H, Ohishi $M$. Marsupialization of cystic ameloblastoma: a clinical and histopathologic study of the growth characteristics before and after marsupialization. J Oral Maxillofac Surg. 1995;53(7):748-754.

15. Lingen MW. Lucas' pathology of tumors of the oral tissues. Arch Pathol Lab Med. 2000;124(3):475.

16. Yang Z, Liang Q, Yang L, Zheng Gs, Zhang Se, Lao $\mathrm{Xm}$, et al. Marsupialization of mandibular cystic ameloblastoma: Retrospective study of 7 years. Head \& Neck. 2018;40(10):2172-2180.

17. Lau SL, Samman N. Recurrence related to treatment modalities of unicystic ameloblastoma: a systematic review. Int J Oral Maxillofac Surg. 2006;35(8):681-690.

18. Gardner DG. A pathologist's approach to the treatment of ameloblastoma J Oral Maxillofac Surg. 1984;42:161166.

19. Corio RL, Goldblatt LI, Edwards PA, Hartman KS. Ameloblastic carcinoma: a clinicopathologic study and assessment of eight cases. Oral Surg Oral Med Oral Pathol. 1987;64(5):570-576. 